\title{
Association between Airborne Fine Particulate Matter and Residents' Cardiovascular Diseases, Ischemic Heart Disease and Cerebral Vascular Disease Mortality in Areas with Lighter Air Pollution in China
}

\author{
Junfang Cai ${ }^{1,+}$, Shuyuan $\mathrm{Yu}^{2,+}$, Yingxin Pei ${ }^{3}$, Chaoqiong Peng ${ }^{2}$, Yuxue Liao ${ }^{2}$, Ning Liu ${ }^{2}$, \\ Jiajia $\mathrm{Ji}^{2}$ and Jinquan Cheng ${ }^{2, *}$ \\ 1 National Institute of Environmental Health and Related Product Safety, Chinese Center for Disease Control \\ and Prevention, Beijing 100021, China; caijunfang@nieh.chinacdc.cn \\ 2 Shenzhen Center for Disease Control and Prevention, Shenzhen 518055, China; \\ shuyuanyu2008@163.com (S.Y.); pcq@szcdc.net (C.P.); lyxchinaren@163.com (Y.L.); liun@szcdc.net (N.L.); \\ jiajia0929@sohu.com (J.J.) \\ 3 CFETP, Chinese Center for Disease Control and Prevention, Beijing 100050, China; peiyingxin@hotmail.com \\ * Correspondence: cjinquan@szcdc.net; Tel.: 86-135-0281-3859 \\ + Co-first authors: Junfang Cai and Shuyuan Yu contributed equally to this work.
}

Received: 5 July 2018; Accepted: 28 August 2018; Published: 3 September 2018

\begin{abstract}
Background: China began to carry out fine particulate matter $\left(\mathrm{PM}_{2.5}\right)$ monitoring in 2013 and the amount of related research is low, especially in areas with lighter air pollution. This study aims to explore the association between $\mathrm{PM}_{2.5}$ and cardiovascular disease (CVD), ischemic heart disease (IHD) and cerebral vascular disease (EVD) mortality in areas with lighter air pollution. Methods: Data on resident mortality, air pollution and meteorology in Shenzhen during 2013-2015 were collected and analyzed using semi-parametric generalized additive models (GAM) with Poisson distribution of time series analysis. Results: Six pollutants were measured at seven air quality monitoring sites, including $\mathrm{PM}_{2.5}, \mathrm{PM}_{10}, \mathrm{SO}_{2}, \mathrm{NO}_{2}, \mathrm{CO}$ and $\mathrm{O}_{3}$. The $\mathrm{PM}_{2.5}$ daily average concentration was 35.0 $\pm 21.9 \mu \mathrm{g} / \mathrm{m}^{3}$; the daily average concentration range was from $7.1 \mu \mathrm{g} / \mathrm{m}^{3}$ to $137.1 \mu \mathrm{g} / \mathrm{m}^{3}$. $\mathrm{PM}_{2.5}$ concentration had significant effects on CVD, IHD and EVD mortality. While $\mathrm{PM}_{2.5}$ concentration of lag5 and lag02 rose by $10 \mu \mathrm{g} / \mathrm{m}^{3}$, the excess risk (ER) of CVD mortality were $1.50 \%$ (95\% CI: $0.51-2.50 \%$ ) and $2.09 \%$ (95\% CI: $0.79-3.41 \%$ ), respectively. While $\mathrm{PM}_{2.5}$ concentration of lag2 and lag02 rose by $10 \mu \mathrm{g} / \mathrm{m}^{3}$, the ER of IHD mortality were 2.87\% (95\% CI: $0.71-5.07 \%$ ) and $3.86 \%$ (95\% CI: $1.17-6.63 \%)$, respectively. While $\mathrm{PM}_{2.5}$ concentration of lag4 and lag04 rose by $10 \mu \mathrm{g} / \mathrm{m}^{3}$, the ER of EVD mortality were 2.09\% (95\% CI: $2.28-3.92 \%$ ) and 3.08\% (95\% CI: $0.68-5.53 \%)$, respectively. Conclusions: $\mathrm{PM}_{2.5}$ increased CVD mortality. The government needs to strengthen the governance of air pollution in areas with a slight pollution.
\end{abstract}

Keywords: air pollution; cardiovascular disease; ischemic heart disease; cerebral vascular disease; mortality; generalized additive model; time-series

\section{Introduction}

The 2006 World Health Organization Air Quality Guidelines recommend using particulate matter with an aerodynamic diameter of under 2.5 microns (fine particulate matter, $\mathrm{PM}_{2.5}$ ), rather than particulate matter with particle size below 10 microns $\left(\mathrm{PM}_{10}\right)$, as an indicator of air particle concentration [1]. Among atmospheric pollutants, fine particulate matter $\left(\mathrm{PM}_{2.5}\right)$ is consistently associated with adverse human health [2-4] and is of great concern to the general public. The majority 
of studies of $\mathrm{PM}_{2.5}$ and daily mortality have been conducted in North America and Europe, with a small number of studies in other regions of the world [5].

Environmental pollution is very serious in China. The Chinese government has paid more and more attention to the prevention and control of environmental pollution. The report of the 19th Session of National Congress of the Communist Party of China regards pollution prevention as one of the three major battles [6]. Governments at all levels attach great importance to environmental pollution control, especially in areas with serious pollution. Lighter polluted areas in China (although the pollution level is much higher than in Europe and America) still do not attract enough attention.

China began to carry out $\mathrm{PM}_{2.5}$ monitoring in 2013, and few studies have focused on this issue, especially in areas with lighter air pollution. "Lighter air pollution" is relative to most parts of China. Both the Chinese Environmental Status Bulletin and the National Air Quality real-time ranking show that Shenzhen has lighter air pollution than other cities in China $[7,8]$. The average annual concentration of $\mathrm{PM}_{2.5}$ in 272 cities in China from 2013 to 2015 nationwide was $56 \mu \mathrm{g} / \mathrm{m}^{3}$; North-east, North, East, Middle-South, South-west and North-west were $52 \mu \mathrm{g} / \mathrm{m}^{3}, 69 \mu \mathrm{g} / \mathrm{m}^{3}, 59 \mu \mathrm{g} / \mathrm{m}^{3}, 56 \mu \mathrm{g} / \mathrm{m}^{3}, 42 \mu \mathrm{g} / \mathrm{m}^{3}$ and $55 \mu \mathrm{g} / \mathrm{m}^{3}$, respectively [9]. At the same time, the $\mathrm{PM}_{2.5}$ annual average concentration in Shenzhen was $35 \mu \mathrm{g} / \mathrm{m}^{3}$. It shows that Shenzhen air pollution is relatively light in China.

Shenzhen is China's first special economic zone. It is the window of China's reform and opening up. It is one of the three largest national financial centers in China. In the "Economist" list of "The Most Economically Competitive Cities in the World" in 2012, Shenzhen ranked second. Shenzhen is situated in South-eastern China, $113^{\circ} 46-114^{\circ} 37 \mathrm{E}$ and $22^{\circ} 27-22^{\circ} 52 \mathrm{~N}$, with an area of $1991.64 \mathrm{~km}^{2}$, neighboring Hong Kong. Shenzhen has a sub-tropical maritime climate (warm temperatures, abundant rainfall). The annual average temperature is $23.5^{\circ} \mathrm{C}$. January and July monthly average temperatures were $16^{\circ} \mathrm{C}$ and $28.9^{\circ} \mathrm{C}$, respectively. This study aims to explore the association between $\mathrm{PM}_{2.5}$ and cardiovascular disease (CVD), ischemic heart disease (IHD) and cerebral vascular disease (EVD) mortality in areas with lighter air pollution in China.

\section{Materials and Methods}

\subsection{Materials}

\subsubsection{Data on Resident Mortality}

Data on resident mortality in Shenzhen during 1 January 2013-31 December 2015 (a total of 1095 days) were collected from Shenzhen Center for Disease Control and Prevention (CDC), Shenzhen Public Security Bureau and Shenzhen Funeral Home. Each record includes a variable identity card number, name, gender, age, date of birth, date of death, the main cause of death and the main cause of death International Classification of Diseases (ICD) coding. Data for the three agencies were compared in accordance with identity card number and name, and repetitive data excluded. There is not one death due to influenza in the database. Grouping the daily mortality of residents and sorting and screening the data according to the International Classification of Diseases Revision 10 (IDC-10), the data encoded as I00-I99 represent total CVD mortality, I20-I25 represent IHD mortality and I60-I69 represent EVD mortality.

\subsubsection{Air Quality Monitoring Data}

There were eleven air quality monitoring sites at the Shenzhen Environmental Monitoring Station. Seven of them were urban air quality monitoring sites. They were Xixiang, Nanyou, Huaqiaocheng, Honghu, Liyuan, Yantian and Longgang. Daily air quality monitoring data were provided by the seven urban air quality monitoring sites, covering the period of 1 January 2013-31 December 2015. The air quality monitoring data were based on the mean of daily average concentration values at the seven sites, including six indicators, namely: $\mathrm{PM}_{2.5}, \mathrm{PM}_{10}$, sulfur dioxide $\left(\mathrm{SO}_{2}\right)$, nitrogen dioxide $\left(\mathrm{NO}_{2}\right)$, carbon monoxide $(\mathrm{CO})$ and ozone $\left(\mathrm{O}_{3}\right)$. The other four were background monitoring sites and 
regional air quality monitoring sites. They were Guanlan, Meisha, Kuiyong and Nanao. Data from these four monitoring sites were not used. The distribution of monitoring sites was shown in Figure 1.

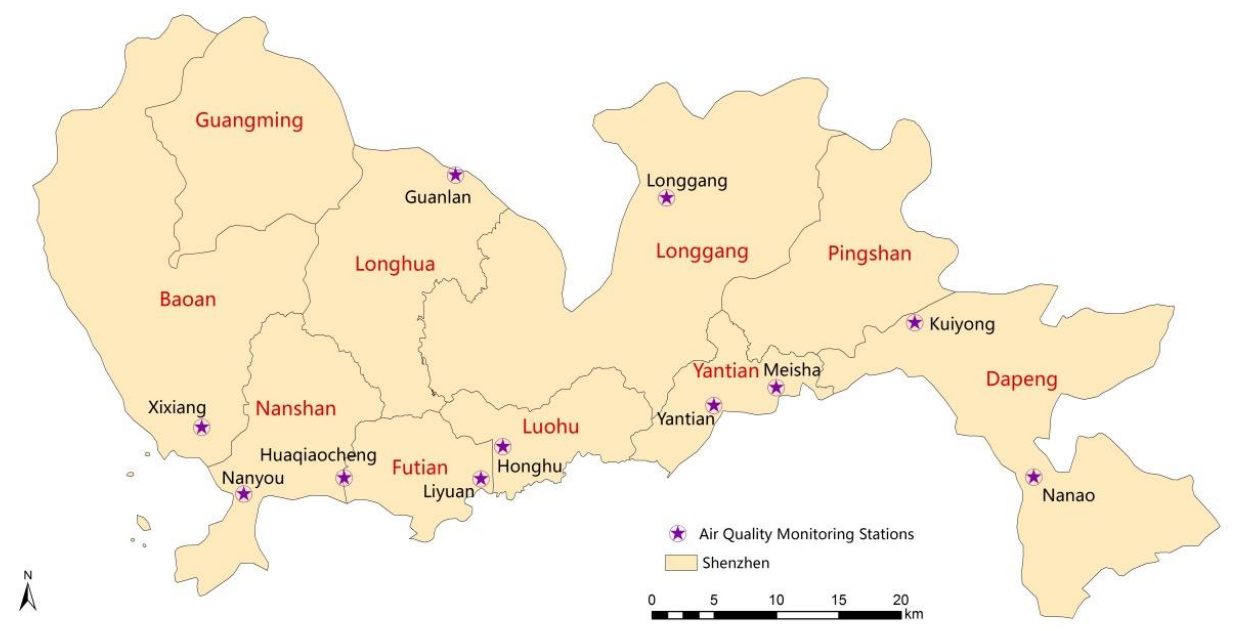

Figure 1. Distribution of state-controlled air quality monitoring sites in Shenzhen.

\subsubsection{Meteorological Data}

There were forty regional meteorological observation stations in Shenzhen. Daily monitoring data from these stations was collected by the Shenzhen Meteorological Service Center. Meteorological data, which was collected from daily routine monitoring data, were released by the Shenzhen Meteorological Service Center, covering the period of 1 January 2013-31 December 2015. This data included daily average temperature, daily average relative humidity $(\mathrm{RH})$, daily average atmospheric pressure and daily average wind speed.

\subsection{Methods}

\subsubsection{Basic Description}

Resident mortality data, daily air pollutant data, and meteorological data were not normally distributed. The data are described by mean, standard deviation, minimum, Q1, Q2, Q3 and maximum in order to show the data more clearly.

\subsubsection{Analysis of Time Series}

Resident mortality data were consolidated into the daily mortality of residents through grouping, screening and summary. The air pollutant variables, $\mathrm{PM}_{2.5}$ and $\mathrm{CO}$, were based on the mean of $24 \mathrm{~h}$ average concentration values at the seven monitoring sites of the Shenzhen Environmental Monitoring Station. The air pollutant $\mathrm{O}_{3}$ variable was based on the means of $8 \mathrm{~h}$ sliding average concentrations. Multiple time series plots were produced with time on the horizontal axis and daily average concentrations of pollutants $\left(\mathrm{PM}_{2.5}, \mathrm{CO}\right.$ and $\left.\mathrm{O}_{3}\right)$ on the vertical coordinate axes.

\subsubsection{Correlation Analysis}

Correlation analysis was conducted using SPSS 19.0 statistical software (SPSS Inc., Chicago, IL, USA). Daily air pollutant data and meteorological data were not normally distributed; therefore, Spearman correlation analysis was conducted with environmental pollutants and meteorological factors. The greater the correlation coefficient between $\mathrm{PM}_{2.5}$ and other factors, the higher the probability of collinearity. In order to control confounding factors and avoid the effects of collinearity, meanwhile considering the effects of $\mathrm{CO}$ and $\mathrm{O}_{3}$ on cardiovascular disease, $\mathrm{CO}$ and $\mathrm{O}_{3}$ were controlled as confounding factors $[10,11]$. 


\subsubsection{Generalized Additive Models (GAM)}

Resident mortality data typically followed a Poisson distribution. Analysis of time series was conducted by using semi-parametric GAM based on Poisson distribution [12-17]. The dependent variable is linked with the independent variable through log transformation [18-20]. Firstly, the basic model was constructed. Following the control of influence of long-term trend, seasonal trend, day of the week and public holiday, daily average temperature and daily average relative humidity were entered into the model $[20,21]$. For the basic model, air pollutants were not entered as independent variables; however, they were used in later models as described in the next paragraph. The long-term trend, daily average temperature and daily average relative humidity were adjusted by cubic regression spline function [22]. Seasonal trend, day of the week and public holiday were adjusted by dummy variables. The degrees of freedom (df) in previous literature were different, ranging from two to ten $[9,23-25]$. Some used the results of previous literature directly [26,27]. The optimal model was fitted to make the result more stable by changing $\mathrm{df}$ to a larger range (the long-term trend $\mathrm{df}$ from one to fourteen per year, the daily average temperature $\mathrm{df}$ from one to seven, and the daily average relative humidity $\mathrm{df}$ from one to seven). Based on the unbiased risk estimate (UBRE, UBRE is a readjustment standard of Akaike information criterion (AIC)) value and previous literature [28,29], the best parameters were established. For the basic regression model, we ultimately used seven df per year for the time variable, three $\mathrm{df}$ for the temperature variable, and three $\mathrm{df}$ for the humidity variable. Then, the single pollutant model was constructed. Single-day lag models underestimate the cumulative effect of pollutants on mortality [30]. Therefore, we built both single-day lag and multi-day lag models. $\mathrm{PM}_{2.5}$ concentration with different lags from 0 to 5 days prior to mortality (lag0-lag5) and moving averages from day 0 to day $1-5$ prior to the mortality (lag01-lag04) were included in the model, respectively. Finally, two-pollutant models were constructed. We did not control $\mathrm{SO}_{2}$ and $\mathrm{NO}_{2}$ in the regression models because our Spearman correlation analysis showed that $\mathrm{PM}_{2.5}$ was highly correlated with $\mathrm{SO}_{2}$ and $\mathrm{NO}_{2}$. Previous literature showed that simultaneously entering $\mathrm{SO}_{2}$ (or $\mathrm{NO}_{2}$ ) into the models can bring unstable parameter estimates when the pollutants involved suffer high inter-correlation [31]. To examine the independent effects of $\mathrm{PM}_{2.5}$ on resident mortality, $\mathrm{CO}$ and $\mathrm{O}_{3}$ were controlled in the regression models respectively because previous literature and our Spearman correlation analysis showed that the correlation between $\mathrm{CO}$ and $\mathrm{PM}_{2.5}$, and $\mathrm{O}_{3}$ and $\mathrm{PM}_{2.5}$, were low [32]. $\mathrm{CO}$ and $\mathrm{O}_{3}$ were included in the regression models respectively with the same lag ( $0-5$ days) prior to mortality (lag0-lag5) and moving averages from day 0 to day $1-5$ prior to the mortality (lag01-lag04). Two-pollutant models were constructed respectively. The daily average concentration of pollutants $\mathrm{PM}_{2.5}, \mathrm{CO}$ and $\mathrm{O}_{3}$ were used as continuous variables to enter the model. The excess risk $\left(E R, E R=\left(e^{\beta^{*} 10}-1\right)\right)$ of resident mortality with different health effects, as well as its $95 \% \mathrm{CI}$ value, were calculated using regression coefficients and standard errors along with the incremental increase of $10 \mu \mathrm{g} / \mathrm{m}^{3}$ in $\mathrm{PM}_{2.5}$ concentration. Quantitative analysis of the short-term effect of $\mathrm{PM}_{2.5}$ upon resident mortality was conducted [21]. The determined model expression is as follows:

$$
\begin{gathered}
\log \left[E\left(y_{t}\right)\right]=\beta X_{t}+s\left(\text { time } d f_{1}\right)+\text { as.factor }(\text { season })+\text { as.factor }(\text { dow })+ \\
\text { as.factor }(\text { holiday })+s\left(\text { temperature, } d f_{2}\right)+s\left(\text { humidity }, d f_{3}\right)+\alpha
\end{gathered}
$$

Here $y_{t}$ represents the number of mortalities at day $t ; E\left(y_{t}\right)$ represents the expected number of mortalities at day $t ; \beta$ is the linear regression coefficients estimated by the GAM; $X_{t}$ indicates the concentration of pollutants at day $t ; s$ represents the nonparametric smoothing function; $d f_{1}$ is the degree of freedom for adjusting long-term trend in the nonparametric function; season is the dummy variable for season; dow and holiday are the dummy variables for day of the week and holiday, respectively, used to control the short-term fluctuations in the number of daily resident mortality; $d f_{2}$ and $d f_{3}$ are the degrees of freedom for adjusting the nonparametric smoothing function daily average temperature and daily average relative humidity; $\alpha$ is the residual error [22]. Resident mortality data, air quality monitoring data and meteorological data have no missing values. The pollutant 
concentration had missing values when analyzing the lag effect. The missing values were processed by deleting the corresponding record. Effect estimates were considered statistically significant if $p<0.05$, and borderline significant if $p<0.10$ [33]. Specific $p$ values are listed. Statistical analysis was done by R3.2.0 software (open source software, Auckland, New Zealand). The GAM was constructed by the penalized splines function of GAM in the mgcv package.

\section{Results}

\subsection{Basic Information of the Death of Residents from CVD}

A total of 14,537 people died due to CVD; 3-32 people died due to CVD every day; the average age of CVD death was 67.4 years old, and the age range was 0-109 years old. In residents that died from CVD, 3,188 people died because of IHD, accounting for $21.9 \%$ of the total CVD deaths; 0-14 people died due to IHD every day; the average age of IHD death was 66.6 years old, and the age range was 0-109 years old. EVD accounted for 4,028 deaths, which was $27.7 \%$ of the total CVD deaths; $0-13$ people died due to EVD every day; the average age of EVD death was 65.9 years old, and the age range was 0-104 years old. See Table 1 for the basic information of death residents from CVD in Shenzhen (2013-2015).

Table 1. Basic information of death residents from cardiovascular disease (CVD) in Shenzhen (2013-2015).

\begin{tabular}{lcccccccc}
\hline Category & $\begin{array}{c}\text { Total } \\
\text { Deaths }\end{array}$ & $\begin{array}{c}\text { Percent } \\
\text { Among Total } \\
\text { Deaths (\%) }\end{array}$ & $\begin{array}{c}\text { Daily Average } \\
\text { Number of } \\
\text { Deaths }\end{array}$ & $\begin{array}{c}\text { Standard } \\
\text { Deviation }\end{array}$ & $\begin{array}{c}\text { Lowest } \\
\text { Daily } \\
\text { Deaths }\end{array}$ & Q1 & Q2 & $\begin{array}{c}\text { Highest } \\
\text { Daily } \\
\text { Deaths }\end{array}$ \\
\hline Total death & 41,815 & 100.0 & 38.2 & 7.6 & 10 & 33 & 38 & 43 \\
CVD death & 14,537 & 34.8 & 13.3 & 4.1 & 3 & 11 & 13 & 16 \\
IHD death & 3188 & 7.6 & 2.9 & 1.8 & 0 & 2 & 3 & 4 \\
EVD death & 4028 & 9.6 & 3.7 & 2.0 & 0 & 2 & 4 & 5 \\
\hline
\end{tabular}

Q1, Q2, Q3 are equal to the $25 \%, 50 \%$ and $75 \%$ number of all values in the sample arranged from small to large, respectively. $\mathrm{CVD}=$ cardiovascular disease. $\mathrm{IHD}=$ ischemic heart disease. $\mathrm{EVD}=$ cerebral vascular disease.

\subsection{Information of Air Pollutants and Meteorological Factors}

In 2013-2015, the $\mathrm{PM}_{2.5}$ annual average concentrations in Shenzhen were $40.2 \mu \mathrm{g} / \mathrm{m}^{3}, 34.7 \mu \mathrm{g} / \mathrm{m}^{3}$ and $30.1 \mu \mathrm{g} / \mathrm{m}^{3}$, respectively; the mean of the $\mathrm{PM}_{2.5}$ daily average concentration was $35.0 \mu \mathrm{g} / \mathrm{m}^{3}$, the daily average concentration range was from $7.1 \mu \mathrm{g} / \mathrm{m}^{3}$ to $137.1 \mu \mathrm{g} / \mathrm{m}^{3}$, exceeding the national Grade 1 criterion of China (concentration limit $<35 \mu \mathrm{g} / \mathrm{m}^{3}$ ) on 458 days [34], and exceeding the national Grade 2 criterion of China (Concentration limit $<75 \mu \mathrm{g} / \mathrm{m}^{3}$ ) on 58 days. $\mathrm{NO}_{2}$ annual average concentration exceeded the national criterion of China (concentration limit $<40 \mu \mathrm{g} / \mathrm{m}^{3}$ ). $\mathrm{PM}_{10}$ and $\mathrm{SO}_{2}$ annual average concentration reached the national Grade 2 (concentration limit: $\mathrm{PM}_{10}<70 \mu \mathrm{g} / \mathrm{m}^{3}, \mathrm{SO}_{2}<60 \mu \mathrm{g} / \mathrm{m}^{3}$ ) criterion and Grade 1 (concentration limit: $\mathrm{PM}_{10}<40 \mu \mathrm{g} / \mathrm{m}^{3}$, $\mathrm{SO}_{2}<20 \mu \mathrm{g} / \mathrm{m}^{3}$ ) criterion of China, respectively. See Table 2 for a summary of air pollutants and meteorological indicators. See Table S1 for People's Republic of China Ministry of Environmental Protection, Atmospheric environmental quality standards (GB3095-2012).

Table 2. Air pollutants and meteorological factors in Shenzhen (2013-2015).

\begin{tabular}{|c|c|c|c|c|c|c|c|c|c|}
\hline Indicator & Mean & $\begin{array}{l}\text { Standard } \\
\text { Deviation }\end{array}$ & Minimum & Q1 & Q2 & Q3 & Maximum & $\begin{array}{l}\text { No. of Days of } \\
\text { Exceeding } \\
\text { Grade } 1 \\
\text { Criterion* }\end{array}$ & $\begin{array}{l}\text { No. of Days of } \\
\text { Exceeding } \\
\text { Grade } 2 \\
\text { Criterion }\end{array}$ \\
\hline \multicolumn{10}{|l|}{ Air pollutants } \\
\hline $\mathrm{PM}_{2.5}\left(\mu \mathrm{g} / \mathrm{m}^{3}\right)$ & 35.0 & 21.9 & 7.1 & 17.4 & 29.9 & 47.1 & 137.1 & 458 & 58 \\
\hline $\mathrm{PM}_{10}\left(\mu \mathrm{g} / \mathrm{m}^{3}\right)$ & 55.6 & 30.2 & 10.9 & 31.4 & 47.7 & 71.6 & 181.8 & 513 & 11 \\
\hline $\mathrm{SO}_{2}\left(\mu \mathrm{g} / \mathrm{m}^{3}\right)$ & 10.4 & 4.8 & 3.5 & 7.4 & 9.2 & 11.9 & 54.8 & 1 & 0 \\
\hline $\mathrm{NO}_{2}\left(\mu \mathrm{g} / \mathrm{m}^{3}\right)$ & 43.8 & 17.5 & 14.7 & 31.9 & 39.7 & 51.6 & 133.7 & 47 & 47 \\
\hline $\mathrm{CO}\left(\mu \mathrm{g} / \mathrm{m}^{3}\right)$ & 1061.9 & 259.7 & 542.9 & 857.1 & 1034.1 & 1239.1 & 1930.4 & 0 & 0 \\
\hline $\mathrm{O}_{3}\left(\mu \mathrm{g} / \mathrm{m}^{3}\right)$ & 55.2 & 22.3 & 16.4 & 37.6 & 50.8 & 70.0 & 143.3 & 42 & 0 \\
\hline
\end{tabular}


Table 2. Cont

\begin{tabular}{|c|c|c|c|c|c|c|c|c|c|}
\hline Indicator & Mean & $\begin{array}{l}\text { Standard } \\
\text { Deviation }\end{array}$ & Minimum & Q1 & Q2 & Q3 & Maximum & $\begin{array}{l}\text { No. of Days of } \\
\text { Exceeding } \\
\text { Grade } 1 \\
\text { Criterion * }\end{array}$ & $\begin{array}{l}\text { No. of Days of } \\
\text { Exceeding } \\
\text { Grade } 2 \\
\text { Criterion }\end{array}$ \\
\hline \multicolumn{10}{|l|}{ Meteorological factors } \\
\hline $\begin{array}{c}\text { Daily average } \\
\text { temperature }\left({ }^{\circ} \mathrm{C}\right)\end{array}$ & 23. 5 & 5.5 & 6.0 & 19.2 & 24.8 & 28.2 & 33.0 & & \\
\hline $\begin{array}{c}\text { Daily average RH (\%) } \\
\text { Daily average }\end{array}$ & 73.3 & 13.3 & 19.0 & 67.0 & 75.0 & 82.0 & 100.0 & & \\
\hline $\begin{array}{c}\text { atmosphere pressure } \\
(\mathrm{kpa})\end{array}$ & 1005.6 & 6.4 & 986.8 & 1000.7 & 1005.5 & 1010.8 & 1019.9 & & \\
\hline $\begin{array}{l}\text { Daily average wind } \\
\text { speed }(\mathrm{m} / \mathrm{s})\end{array}$ & 2.1 & 0.8 & 0.3 & 1.5 & 2.0 & 2.5 & 5.5 & & \\
\hline
\end{tabular}

Q1, Q2, Q3 are equal to the $25 \%, 50 \%$ and $75 \%$ number of all values in the sample arranged from small to large, respectively. * The number of days for average daily concentration exceeded the national Grade 1 criterion of China. ${ }^{* *}$ The number of days for average daily concentration exceeded the national Grade 2 criterion of China. The formula for calculating the standard deviation of pollutants is as follows: $\sigma=\sqrt{\frac{1}{N} \sum_{i=1}^{N}\left(x_{i}-\mu\right)^{2}}$ Standard Deviation is represented by $\sigma \cdot x_{i}$ represents the average concentration of seven air quality monitoring sites on a given day. $\mu$ indicates the daily average concentration of seven air quality monitoring sites in three years. $N=1095$. $\mathrm{PM}_{2.5}$ = particulate matter with a particle size below 2.5 microns. $\mathrm{PM}_{10}=$ particulate matter with particle size below 10 microns. $\mathrm{RH}=$ relative humidity.

\subsection{Time Series Chart on Resident Mortality from CVD versus Air Pollutant Concentration}

As revealed by the time series chart on resident mortality from CVD in Shenzhen versus air pollutant concentration in 2013-2015, the variation trend of CVD death and $\mathrm{PM}_{2.5}$ concentration was basically the same; both peaked in winter and spring, and both declined in summer and autumn. CO and $\mathrm{O}_{3}$ also have seasonal trends. See Figure 2 for details.
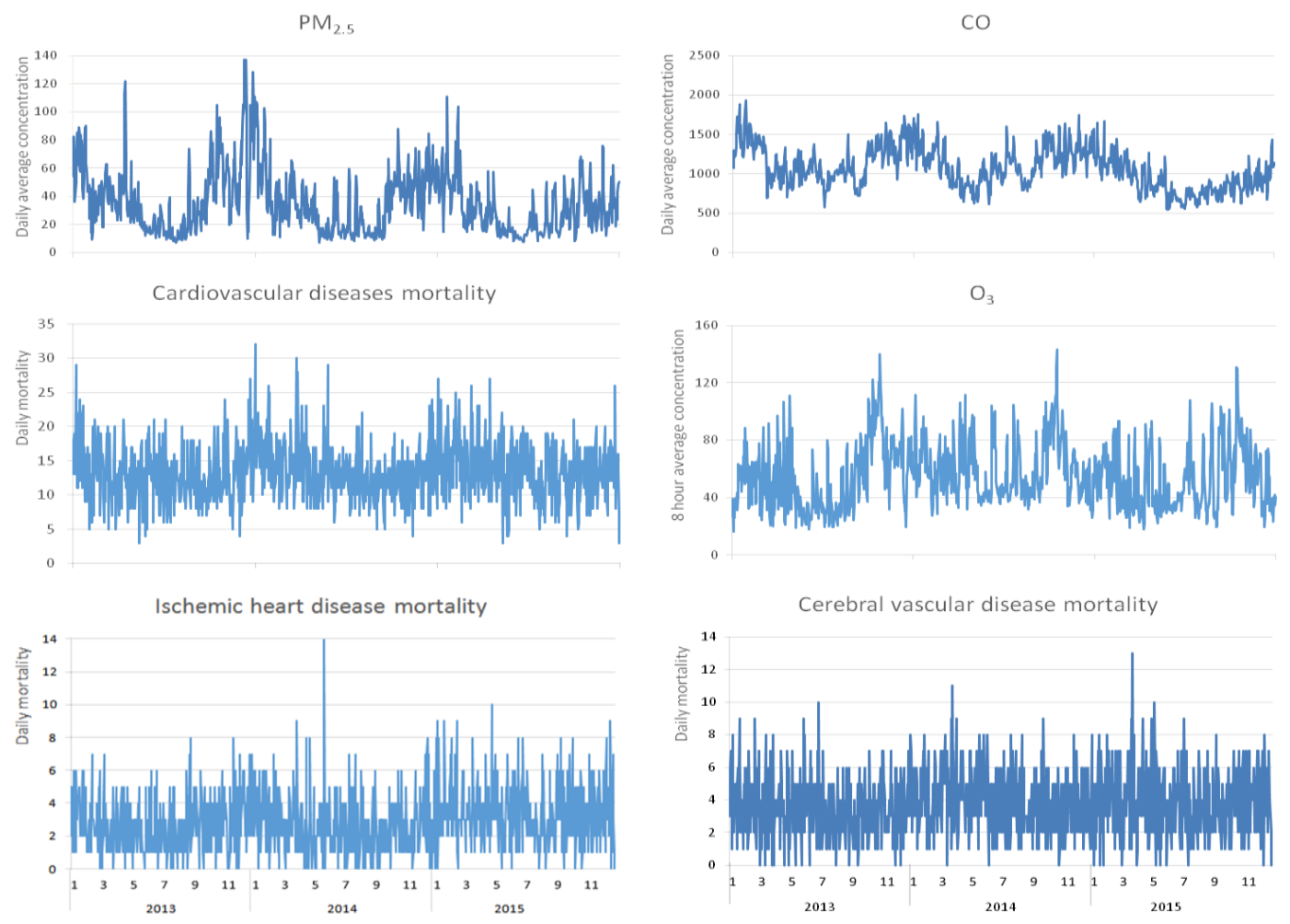

Figure 2. Time series chart on resident mortality versus air pollutant concentration in Shenzhen (2013-2015). 


\subsection{Correlation Analysis of Air Pollutants versus Meteorological Factors}

According to the findings of the Spearman correlation analysis of air pollutants versus meteorological factors, among the six air pollutants, $\mathrm{PM}_{2.5}, \mathrm{PM}_{10}, \mathrm{SO}_{2}, \mathrm{NO}_{2}, \mathrm{CO}$ and $\mathrm{O}_{3}$ exhibited positive correlations between each pair $(p<0.05)$. The correlation between $\mathrm{PM}_{2.5}$ and $\mathrm{PM}_{10}$ was the strongest; their correlation coefficient was more than 0.9. Following that, the correlation between $\mathrm{PM}_{2.5}$ and $\mathrm{SO}_{2}$, and $\mathrm{PM}_{2.5}$ and $\mathrm{NO}_{2}$, were the next strongest. Their correlation coefficients were more than 0.6, respectively. The correlation between $\mathrm{PM}_{2.5}$ and $\mathrm{CO}$, and $\mathrm{PM}_{2.5}$ and $\mathrm{O}_{3}$, were the weakest, and their correlation coefficients were 0.4 and 0.6 , respectively. In terms of air pollutants versus meteorological factors, $\mathrm{PM}_{2.5}, \mathrm{PM}_{10}, \mathrm{SO}_{2}$ and $\mathrm{NO}_{2}$ each exhibited negative correlations with daily average temperature, daily average $\mathrm{RH}$ and daily average wind speed; $\mathrm{PM}_{2.5}, \mathrm{PM}_{10}, \mathrm{SO}_{2}$ and $\mathrm{NO}_{2}$ each exhibited positive correlations with daily average atmosphere pressure. A positive correlation was observed between daily average temperature and daily average $\mathrm{RH}$ (Table 3.)

Table 3. Spearman correlation analysis of air pollutants and meteorological factors in Shenzhen during 2013-2015 (r value).

\begin{tabular}{|c|c|c|c|c|c|c|c|c|c|}
\hline Items & $\mathrm{PM}_{10}$ & $\mathrm{SO}_{2}$ & $\mathrm{NO}_{2}$ & $\mathrm{CO}$ & $\mathrm{O}_{3}$ & $\begin{array}{c}\text { Daily } \\
\text { Average } \\
\text { Temperature }\end{array}$ & $\begin{array}{c}\text { Daily } \\
\text { Average } \\
\text { RH }\end{array}$ & $\begin{array}{c}\text { Daily } \\
\text { Average } \\
\text { Atmosphere } \\
\text { Pressure }\end{array}$ & $\begin{array}{c}\text { Daily } \\
\text { Average } \\
\text { Wind } \\
\text { Speed }\end{array}$ \\
\hline $\mathrm{PM}_{2.5}$ & 0.956 & 0.622 & 0.628 & 0.447 & 0.567 & -0.556 & -0.515 & 0.595 & -0.111 \\
\hline $\mathrm{PM}_{10}$ & & 0.680 & 0.628 & 0.399 & 0.601 & -0.447 & -0.591 & 0.529 & -0.127 \\
\hline $\mathrm{SO}_{2}$ & & & 0.612 & 0.257 & 0.318 & -0.176 & -0.515 & 0.270 & -0.143 \\
\hline $\mathrm{NO}_{2}$ & & & & 0.343 & $0.063 *$ & -0.415 & -0.118 & 0.346 & -0.400 \\
\hline $\mathrm{CO}$ & & & & & 0.189 & -0.315 & $-0.075^{*}$ & 0.242 & $0.037 \mathrm{n}$ \\
\hline $\mathrm{O}_{3}$ & & & & & & $-0.117 \mathrm{n}$ & -0.572 & 0.240 & $0.078 *$ \\
\hline $\begin{array}{c}\text { Daily average } \\
\text { temperature }\left({ }^{\circ} \mathrm{C}\right)\end{array}$ & & & & & & & 0.200 & -0.858 & -0.093 \\
\hline Daily average RH (\%) & & & & & & & & -0.451 & $-0.046 n$ \\
\hline $\begin{array}{c}\text { Daily average } \\
\text { atmosphere pressure } \\
(\mathrm{kpa})\end{array}$ & & & & & & & & & $0.047 \mathrm{n}$ \\
\hline
\end{tabular}

$p<0.01$, except $^{*} p<0.05$ and $\mathrm{n} p>0.05$.

\subsection{Autocorrelation between Different Lags for $P M_{2.5}$}

There was autocorrelation between different lags of $\mathrm{PM}_{2.5}$. According to the findings of the autocorrelation between different lags for $\mathrm{PM}_{2.5}$, the correlation was strongest with a one day interval. The longer the interval, the weaker the correlation. The correlation coefficient was 0.825 at the maximum and 0.531 at the minimum. See Table 4 for details.

Table 4. Correlation coefficient of different lags for $\mathrm{PM}_{2.5}\left(\mu \mathrm{g} / \mathrm{m}^{3}\right)$.

\begin{tabular}{cccccc}
\hline Items & lag1 & lag2 & lag3 & lag4 & lag5 \\
\hline lag0 & 0.825 & 0.692 & 0.631 & 0.582 & 0.531 \\
lag1 & & 0.825 & 0.692 & 0.631 & 0.583 \\
lag2 & & 0.824 & 0.691 & 0.631 \\
lag3 & & & 0.825 & 0.691 \\
lag4 & & & & 0.824 \\
\hline $\mathrm{PM}_{2.5}$ concentration with different lags from 0 to 5 days prior to mortality (lag0-lag5).
\end{tabular}

\subsection{Analysis of GAM}

For the effects of $\mathrm{PM}_{2.5}$ concentration on CVD mortality, IHD mortality and EVD mortality without controlling other pollutants and after controlling $\mathrm{CO}$ or $\mathrm{O}_{3}$, see Table 5 . 
Table 5. The effects of $\mathrm{PM}_{2.5}$ concentration on CVD, IHD and EVD mortality without controlling other pollutants and controlling CO or $\mathrm{O}_{3}$ in Shenzhen (2013-2015).

\begin{tabular}{|c|c|c|c|c|c|c|c|c|c|c|c|c|}
\hline \multirow{2}{*}{ Items } & \multicolumn{4}{|c|}{ Single Pollutant Model (Without Controlling Other Pollutants) } & \multicolumn{4}{|c|}{ 2-Pollutant Model (Controlling CO) } & \multicolumn{4}{|c|}{ 2-Pollutant Model (Controlling $\mathrm{O}_{3}$ ) } \\
\hline & $\beta$ & StdErr & $P$ & ER\% $(95 \% \mathrm{CI})$ & $\beta$ & StdErr & $P$ & ER\% $(95 \% \mathrm{CI})$ & $\beta$ & StdErr & $P$ & ER $(95 \%$ CI $)$ \\
\hline \multicolumn{13}{|c|}{ Cardiovascular disease mortality } \\
\hline $\operatorname{lag} 0$ & 0.0016 & 0.0006 & $0.005 \#$ & $1.60(0.48-2.73)$ & 0.0018 & 0.0006 & $0.005 \#$ & $1.83(0.54-3.13)$ & 0.0016 & 0.0006 & $0.007 \#$ & $1.63(0.45-2.82)$ \\
\hline $\operatorname{lag} 1$ & 0.0014 & 0.0005 & $0.010 *$ & $1.40(0.33-2.49)$ & 0.0016 & 0.0006 & $0.006 \#$ & $1.65(0.46-2.86)$ & 0.0016 & 0.0006 & $0.008 \#$ & $1.58(0.41-2.76)$ \\
\hline lag2 & 0.0014 & 0.0005 & $0.010 *$ & $1.37(0.33-2.42)$ & 0.0016 & 0.0006 & $0.005 \#$ & $1.65(0.51-2.82)$ & 0.0015 & 0.0006 & $0.009 \#$ & $1.55(0.39-2.73)$ \\
\hline lag3 & 0.0010 & 0.0005 & $0.053 @$ & $1.00(-0.01-2.02)$ & 0.0011 & 0.0006 & $0.042 *$ & $1.15(0.04-2.28)$ & 0.0010 & 0.0006 & $0.074 @$ & $1.04(-0.1-2.2)$ \\
\hline $\operatorname{lag} 4$ & 0.0009 & 0.0005 & $0.081 @$ & $0.89(-0.11-1.90)$ & 0.0012 & 0.0006 & $0.034 *$ & $1.19(0.09-2.31)$ & 0.0012 & 0.0006 & $0.031 *$ & $1.25(0.12-2.4)$ \\
\hline lag5 & 0.0015 & 0.0005 & $0.003 \#$ & $1.50(0.51-2.50)$ & 0.0016 & 0.0006 & $0.004 \#$ & $1.61(0.52-2.72)$ & 0.0023 & 0.0006 & $0.000 \#$ & $2.28(1.15-3.42)$ \\
\hline lag01 & 0.0018 & 0.0006 & $0.003 \#$ & $1.85(0.63-3.09)$ & 0.0021 & 0.0007 & $0.002 \#$ & $2.1(0.75-3.48)$ & 0.0020 & 0.0007 & $0.003 \#$ & $2.04(0.7-3.39)$ \\
\hline lag02 & 0.0021 & 0.0007 & $0.002 \#$ & $2.09(0.79-3.41)$ & 0.0023 & 0.0007 & $0.001 \#$ & $2.37(0.96-3.81)$ & 0.0024 & 0.0007 & $0.001 \#$ & $2.45(0.98-3.93)$ \\
\hline lag03 & 0.0021 & 0.0007 & $0.002 \#$ & $2.14(0.79-3.51)$ & 0.0024 & 0.0007 & $0.001 \#$ & $2.39(0.93-3.86)$ & 0.0025 & 0.0008 & $0.001 \#$ & $2.56(1-4.14)$ \\
\hline lag04 & 0.0021 & 0.0007 & $0.002 \#$ & $2.17(0.78-3.59)$ & 0.0024 & 0.0008 & $0.001 \#$ & $2.43(0.93-3.95)$ & 0.0027 & 0.0008 & $0.001 \#$ & $2.77(1.14-4.43)$ \\
\hline \multicolumn{13}{|c|}{ Ischemic heart disease mortality } \\
\hline $\operatorname{lag} 0$ & 0.0021 & 0.0012 & $0.072 @$ & $2.12(-0.18-4.47)$ & 0.0032 & 0.0014 & $0.018 *$ & $3.26(0.56-6.03)$ & 0.0026 & 0.0012 & $0.035 *$ & $2.62(0.19-5.11)$ \\
\hline lag1 & 0.0029 & 0.0011 & 0.010 * & $2.92(0.69-5.20)$ & 0.0042 & 0.0013 & $0.001 \#$ & $4.3(1.76-6.91)$ & 0.0033 & 0.0012 & $0.006 \#$ & $3.36(0.93-5.85)$ \\
\hline lag2 & 0.0028 & 0.0011 & $0.009 \#$ & $2.87(0.71-5.07)$ & 0.0043 & 0.0012 & $0.000 \#$ & $4.38(1.93-6.88)$ & 0.0031 & 0.0012 & 0.010 * & $3.14(0.74-5.61)$ \\
\hline lag3 & 0.0011 & 0.0011 & 0.289 & $1.12(-0.94-3.24)$ & 0.0020 & 0.0012 & $0.091 @$ & $2.01(-0.32-4.39)$ & 0.0008 & 0.0012 & 0.487 & $0.84(-1.5-3.23)$ \\
\hline lag4 & 0.0014 & 0.0010 & 0.179 & $1.41(-0.64-3.50)$ & 0.0020 & 0.0012 & $0.089 @$ & $2(-0.3-4.35)$ & 0.0019 & 0.0012 & 0.104 & $1.94(-0.4-4.34)$ \\
\hline lag5 & 0.0023 & 0.0010 & $0.023 *$ & $2.37(0.33-4.46)$ & 0.0026 & 0.0012 & $0.022 *$ & $2.68(0.39-5.03)$ & 0.0033 & 0.0012 & $0.005 \#$ & $3.33(0.98-5.73)$ \\
\hline lag01 & 0.0031 & 0.0013 & $0.014 *$ & $3.15(0.63-5.75)$ & 0.0044 & 0.0014 & $0.002 \#$ & $4.55(1.67-7.5)$ & 0.0038 & 0.0014 & $0.006 \#$ & $3.85(1.1-6.68)$ \\
\hline lag02 & 0.0038 & 0.0013 & $0.005 \#$ & $3.86(1.17-6.63)$ & 0.0053 & 0.0015 & $0.000 \#$ & $5.39(2.38-8.49)$ & 0.0046 & 0.0015 & $0.002 \#$ & $4.71(1.68-7.83)$ \\
\hline $\operatorname{lag} 03$ & 0.0035 & 0.0014 & $0.011 *$ & $3.60(0.82-6.45)$ & 0.0050 & 0.0015 & $0.001 \#$ & $5.08(2.01-8.25)$ & 0.0042 & 0.0016 & $0.008 \#$ & $4.34(1.14-7.64)$ \\
\hline lag04 & 0.0035 & 0.0014 & 0.014 * & $3.58(0.73-6.51)$ & 0.0049 & 0.0016 & $0.002 \#$ & $4.98(1.84-8.22)$ & 0.0045 & 0.0017 & $0.007 \#$ & $4.55(1.21-8.01)$ \\
\hline \multicolumn{13}{|c|}{ Cerebral vascular disease mortality } \\
\hline $\operatorname{lag} 0$ & 0.0020 & 0.0010 & $0.045^{*}$ & $2.03(0.04-4.06)$ & 0.0021 & 0.0011 & $0.062 @$ & $2.16(-0.1-4.47)$ & 0.0017 & 0.0011 & 0.121 & $1.67(-0.44-3.81)$ \\
\hline lag1 & 0.0020 & 0.0010 & $0.034 *$ & $2.07(0.16-4.02)$ & 0.0019 & 0.0011 & $0.068 @$ & $1.95(-0.14-4.1)$ & 0.0018 & 0.0011 & $0.092 @$ & $1.81(-0.29-3.96)$ \\
\hline lag2 & 0.0015 & 0.0009 & 0.126 & $1.46(-0.40-3.36)$ & 0.0013 & 0.0010 & 0.199 & $1.34(-0.7-3.42)$ & 0.0017 & 0.0011 & 0.108 & $1.74(-0.38-3.91)$ \\
\hline lag3 & 0.0019 & 0.0009 & $0.040 *$ & $1.90(0.08-3.75)$ & 0.0018 & 0.0010 & $0.080 @$ & $1.78(-0.21-3.81)$ & 0.0021 & 0.0011 & $0.049 *$ & $2.09(0-4.23)$ \\
\hline $\operatorname{lag} 4$ & 0.0021 & 0.0009 & $0.023 *$ & $2.09(0.28-3.92)$ & 0.0025 & 0.0010 & $0.014 *$ & $2.49(0.5-4.51)$ & 0.0018 & 0.0011 & $0.085 @$ & $1.83(-0.25-3.95)$ \\
\hline lag5 & 0.0020 & 0.0009 & $0.029 *$ & $1.99(0.20-3.81)$ & 0.0020 & 0.0010 & $0.043 *$ & $2.03(0.07-4.04)$ & 0.0018 & 0.0010 & $0.083 @$ & $1.83(-0.23-3.94)$ \\
\hline lag01 & 0.0024 & 0.0011 & $0.024 *$ & $2.46(0.32-4.64)$ & 0.0024 & 0.0012 & $0.043 *$ & $2.43(0.07-4.84)$ & 0.0020 & 0.0012 & $0.085 @$ & $2.06(-0.28-4.47)$ \\
\hline lag02 & 0.0025 & 0.0011 & $0.028 *$ & $2.52(0.27-4.82)$ & 0.0024 & 0.0012 & $0.051 @$ & $2.43(-0.01-4.93)$ & 0.0024 & 0.0013 & $0.064 @$ & $2.43(-0.14-5.06)$ \\
\hline lag03 & 0.0027 & 0.0012 & $0.019 *$ & $2.78(0.45-5.16)$ & 0.0026 & 0.0013 & $0.039 *$ & $2.63(0.13-5.19)$ & 0.0028 & 0.0014 & $0.043 *$ & $2.82(0.09-5.62)$ \\
\hline lag04 & 0.0030 & 0.0012 & $0.012 *$ & $3.08(0.68-5.53)$ & 0.0029 & 0.0013 & $0.022 *$ & $2.99(0.43-5.61)$ & 0.0029 & 0.0014 & $0.041 *$ & $2.97(0.12-5.91)$ \\
\hline
\end{tabular}

$\# p<0.01 ; * p<0.05 ; @ p<0.10$. StdErr $=$ standard error. ER = excess risk. 


\subsubsection{Effects of $\mathrm{PM}_{2.5}$ Concentration upon CVD Mortality}

Without controlling other pollutants, $\mathrm{PM}_{2.5}$ concentration of lag0-lag5 on CVD mortality had significant effects. When $\mathrm{PM}_{2.5}$ concentration of lag0 to lag5 rose by $10 \mu \mathrm{g} / \mathrm{m}^{3}$, the excess risks of CVD mortality were $1.60 \%$ (95\% CI: $0.48-2.73 \%), 1.40 \%$ (95\% CI: $0.33-2.49 \%), 1.37 \%$ (95\% CI: $0.33-2.42 \%), 1.00 \%$ (95\% CI: $-0.01-2.02 \%), 0.89 \%(95 \%$ CI: $-0.11-1.90 \%)$ and $1.50 \%(95 \%$ CI: $0.51-2.50 \%)$, respectively. Of these, lag5 was the most significant $(p=0.003)$; lag3 and lag4 were marginally significant $(p=0.053 ; p=0.081)$. The $\mathrm{PM}_{2.5}$ moving average concentration (lag01-lag04) on CVD mortality had significant effects. When $\mathrm{PM}_{2.5}$ concentration of lag01 to lag04 rose by $10 \mu \mathrm{g} / \mathrm{m}^{3}$, the ER of CVD mortality were $1.85 \%$ (95\% CI: $0.63-3.09 \%$ ), 2.09\% (95\% CI: $0.79-3.41 \%$ ), $2.14 \%$ (95\% CI: $0.79-3.51 \%$ ) and $2.17 \%$ (95\% CI: $0.78-3.59 \%)$, respectively. Of these, lag02 was the most significant $(p=0.0015)$, and all $P$ were less than 0.01 . After controlling CO, the results show that $\mathrm{PM}_{2.5}$ concentration of lag0-lag5 on CVD mortality still had significant effects. When $\mathrm{PM}_{2.5}$ concentration of lag0 to lag5 rose by $10 \mu \mathrm{g} / \mathrm{m}^{3}$, the ER of CVD mortality were $1.83 \%(95 \% \mathrm{CI}$ : $0.54-3.13 \%), 1.65 \%$ (95\% CI: $0.46-2.86 \%$ ), $1.65 \%$ (95\% CI: 0.51-2.82\%), $1.51 \%$ (95\% CI: $0.04-2.28 \%), 1.19 \%$ (95\% CI: $0.09-2.31 \%$ ) and $1.61 \%$ (95\%CI: $0.52-2.72 \%)$, respectively. Of these, lag5 was still the most significant $(p=0.004)$. The effects maintain stability after controlling CO. The $\mathrm{PM}_{2.5}$ moving average concentration (lag01-lag04) on CVD mortality had significant effects. When $\mathrm{PM}_{2.5}$ concentration of lag01 to lag04 rose by $10 \mu \mathrm{g} / \mathrm{m}^{3}$, the ER of CVD mortality were $2.10 \%$ (95\% CI: $0.75-3.48 \%$ ), $2.37 \%$ (95\% CI: $0.96-3.81 \%), 2.39 \%$ (95\% CI: $0.93-3.86 \%$ ) and $2.43 \%$ (95\% CI: $0.93-3.95 \%)$, respectively. Of these, lag02 was the most significant $(p=0.0010)$, and all $P$ were less than 0.01 . The effects maintain stability after controlling $\mathrm{CO}$. After controlling $\mathrm{O}_{3}$, the results show that $\mathrm{PM}_{2.5}$ concentration of lag0-lag5 on CVD mortality still had significant effects. When $\mathrm{PM}_{2.5}$ concentration of lag0 to lag5 rose by $10 \mu \mathrm{g} / \mathrm{m}^{3}$, the ER of CVD mortality were 1.63\% (95\% CI: 0.45-2.82\%), 1.58\% (95\% CI: $0.41-2.76 \%), 1.55 \%$ (95\% CI: $0.39-2.73 \%), 1.04 \%$ (95\% CI: $-0.10-2.20 \%), 1.25 \%$ (95\% CI: $0.12-2.40 \%$ ) and $2.28 \%$ (95\% CI: $1.15-3.42 \%)$, respectively. Of these, lag5 was still the most significant $(p=0.000)$. The effects maintain stability after controlling $\mathrm{O}_{3}$. The $\mathrm{PM}_{2.5}$ moving average concentration (lag01-lag04) on CVD mortality had significant effects. When $\mathrm{PM}_{2.5}$ concentration of lag01 to lag04 rose by $10 \mu \mathrm{g} / \mathrm{m}^{3}$, the ER of CVD mortality were $2.04 \%$ (95\% CI: $0.70-3.39 \%$ ), $2.45 \%$ (95\% CI: $0.98-3.93 \%$ ), $2.56 \%$ (95\% CI: $1.00-4.14 \%$ ) and $2.77 \%$ (95\% CI: $1.14-4.43 \%)$, respectively. Of these, lag02 was the most significant $(p=0.0010)$, and all $P$ were less than 0.01 . The effects maintain stability after controlling $\mathrm{O}_{3}$.

\subsubsection{Effects of $\mathrm{PM}_{2.5}$ Concentration upon IHD Mortality}

Without controlling other pollutants, $\mathrm{PM}_{2.5}$ concentration of lag 0 to lag2 and lag 5 on IHD mortality had significant effects. When $\mathrm{PM}_{2.5}$ concentration of lag0 to lag2 and lag5 rose by $10 \mu \mathrm{g} / \mathrm{m}^{3}$, the ER of IHD mortality were $2.12 \%$ (95\% CI: $-0.18-4.47 \%), 2.92 \%$ (95\% CI: $0.69-5.20 \%), 2.87 \%$ (95\% CI: $0.71-5.07 \%)$ and $2.37 \%$ (95\% CI: $0.33-4.46 \%)$, respectively. Of these, lag2 was the most significant $(p=0.009)$ and lag0 was marginally significant $(p=0.072)$. The $\mathrm{PM}_{2.5}$ moving average concentration (lag01-lag04) on IHD mortality had significant effects. When $\mathrm{PM}_{2.5}$ concentration of lag01 to lag04 rose by $10 \mu \mathrm{g} / \mathrm{m}^{3}$, the ER of IHD mortality were $3.51 \%$ (95\% CI: $0.63-5.75 \%$ ), 3.86\% (95\% CI: $1.17-6.63 \%$ ), $3.60 \%$ (95\% CI: $0.82-6.64 \%$ ) and 3.58\% (95\% CI: $0.73-6.51 \%)$, respectively. Of these, lag02 was the most significant $(p=0.005)$. After controlling $\mathrm{CO}$, the results show that $\mathrm{PM}_{2.5}$ concentration of lag0-lag5 on IHD mortality still had significant effects. When $\mathrm{PM}_{2.5}$ concentration of lag0 to lag 5 rose by $10 \mu \mathrm{g} / \mathrm{m}^{3}$, the ER of IHD mortality were 3.26\% (95\% CI: 0.56-6.03\%), 4.30\% (95\% CI:1.76-6.91\%), $4.38 \%$ (95\% CI: $1.93-6.88 \%$ ), $2.01 \%$ (95\% CI: $-0.32-4.39 \%), 2 \%(95 \%$ CI: $-0.30-4.35 \%)$ and $2.68 \%$ (95\% CI: $0.39-5.03 \%)$, respectively. Of these, lag2 was still the most significant $(p=0.000)$ and lag3 and lag4 were marginally significant $(p=0.091 ; p=0.089)$. The $\mathrm{PM}_{2.5}$ moving average concentration (lag01-lag04) on IHD mortality had significant effects. When $\mathrm{PM}_{2.5}$ concentration of lag01 to lag04 rose by $10 \mu \mathrm{g} / \mathrm{m}^{3}$, the ER of IHD mortality were 4.55\% (95\% CI: 1.67-7.50\%), 5.39\% (95\% CI: $2.38-8.49 \%), 5.08 \%$ (95\% CI: $2.01-8.25 \%)$ and $4.98 \%$ (95\% CI: $1.84-8.22 \%)$, respectively. Of these, lag02 was the most significant $(p=0.000)$, and all $P$ were less than 0.01 . The effects maintain stability after controlling CO. After 
controlling $\mathrm{O}_{3}$, the results still show that $\mathrm{PM}_{2.5}$ concentration of lag0 to lag2 and lag5 on IHD mortality had significant effects. When $\mathrm{PM}_{2.5}$ concentration of lag0 to lag2 and lag5 rose by $10 \mu \mathrm{g} / \mathrm{m}^{3}$, the ER of IHD mortality were 2.62\% (95\% CI: $0.19-5.11 \%), 3.36 \%$ (95\% CI: $0.93-5.85 \%), 3.14 \%$ (95\% CI: $0.74-5.61 \%), 3.33 \%$ (95\% CI: $0.98-5.73 \%)$, respectively. Of these, lag5 was still the most significant $(p=0.005)$. The $\mathrm{PM}_{2.5}$ moving average concentration (lag01-lag04) on IHD mortality had significant effects. When $\mathrm{PM}_{2.5}$ concentration of lag01 to lag04 rose by $10 \mu \mathrm{g} / \mathrm{m}^{3}$, the ER of IHD mortality were $3.85 \%$ (95\% CI: $1.10-6.68 \%$ ), $4.71 \%$ (95\% CI: $1.68-7.83 \%), 4.34 \%$ (95\% CI: $1.14-7.64 \%$ ) and $4.55 \%$ (95\% CI: $1.21-8.01 \%)$, respectively. Of these, lag02 was the most significant $(p=0.002)$, and all $P$ were less than 0.01 . The effects maintain stability after controlling $\mathrm{O}_{3}$.

\subsubsection{Effects of $\mathrm{PM}_{2.5}$ Concentration upon EVD Mortality}

Without controlling other pollutants, $\mathrm{PM}_{2.5}$ concentration of lag0, lag1 and lag3 to lag5 on EVD mortality had significant effects. When $\mathrm{PM}_{2.5}$ concentration of lag0, lag1 and lag3 to lag5 rose by $10 \mathrm{\mu g} / \mathrm{m}^{3}$, the ER of EVD mortality were $2.03 \%$ (95\% CI: $0.04-4.06 \%$ ), $2.07 \%$ (95\% CI: $0.16-4.02 \%$ ), $1.90 \%$ (95\% CI: $0.08-3.75 \%$ ), $2.09 \%$ (95\% CI: 2.28-3.92\%) and 1.99\% (95\% CI: $0.20-3.81 \%$ ), respectively. Of these, lag4 was the most significant $(p=0.023)$. The $\mathrm{PM}_{2.5}$ moving average concentration (lag01-lag04) on EVD mortality had significant effects. When $\mathrm{PM}_{2.5}$ concentration of lag01 to lag04 rose by $10 \mu \mathrm{g} / \mathrm{m}^{3}$, the ER of EVD mortality were $2.46 \%$ (95\% CI: $0.32-4.64 \%$ ), $2.52 \%$ (95\% CI: $0.27-4.82 \%$ ), $2.78 \%$ (95\% CI: $0.45-5.16 \%$ ) and $3.08 \%$ (95\% CI: $0.68-5.53 \%)$, respectively. Of these, lag04 was the most significant $(p=0.012)$. After controlling $\mathrm{CO}$, the results show that $\mathrm{PM}_{2.5}$ concentration of lag0, lag1 and lag3 to lag5 on EVD mortality still had significant effects. When $\mathrm{PM}_{2.5}$ concentration of lag0, lag1 and lag3 to lag5 rose by $10 \mu \mathrm{g} / \mathrm{m}^{3}$, the ER of EVD mortality were $2.16 \%$ (95\% CI: $-0.10-4.47 \%$ ), $1.95 \%$ (95\% CI: $-0.14-4.10 \%), 1.78 \%$ (95\% CI: $-0.21-3.81 \%), 2.49 \%$ (95\% CI: $0.50-4.51 \%$ ) and $2.03 \%$ (95\% CI: $0.07-4.04 \%)$, respectively. Of these, lag4 was still the most significant $(p=0.014)$ and lag0, lag1 and lag3 were marginally significant $\left(p=0.062 ; p=0.068 ; p=0.080\right.$ ). The $\mathrm{PM}_{2.5}$ moving average concentration (lag01-lag04) on EVD mortality had significant effects. When $\mathrm{PM}_{2.5}$ concentration of lag01 to lag04 rose by $10 \mu \mathrm{g} / \mathrm{m}^{3}$, the ER of EVD mortality were $2.43 \%$ (95\% CI: $0.07-4.84 \%$ ), $2.43 \%$ (95\%CI: $-0.01-4.93 \%$ ), $2.63 \%$ (95\%CI: $0.13-5.19 \%$ ) and $2.99 \%$ (95\% CI: 0.43-5.61\%), respectively. Of these, lag04 was the most significant $(p=0.022)$. The effects maintain stability after controlling CO. The $\mathrm{PM}_{2.5}$ moving average concentration (lag02) was marginally significant $(p=0.051)$. After controlling $\mathrm{O}_{3}$, the results show that $\mathrm{PM}_{2.5}$ concentration of lag1 and lag3 to lag5 on EVD mortality had significant effects. When $\mathrm{PM}_{2.5}$ concentration of lag1 and lag3 to lag5 rose by $10 \mu \mathrm{g} / \mathrm{m}^{3}$, the ER of EVD mortality were $1.81 \%$ (95\% CI: $-0.29-3.96 \%), 2.09 \%$ (95\% CI: $0.00-4.23 \%), 1.83 \%$ (95\% CI: $-0.25-3.95 \%), 1.83 \%$ (95\% CI: $-0.23-3.94 \%)$, respectively. Of these, lag3 was still the most significant $(p=0.049)$ and lag1, lag4 and lag5 were marginally significant $(p=0.092 ; p=0.085 ; p=0.083)$. The $\mathrm{PM}_{2.5}$ moving average concentration (lag01-lag04) on EVD mortality had still significant effects. The effects maintain stability after controlling $\mathrm{O}_{3}$. When $\mathrm{PM}_{2.5}$ concentration of lag01 to lag04 rose by $10 \mu \mathrm{g} / \mathrm{m}^{3}$, the ER of EVD mortality were $2.06 \%$ (95\% CI: $-0.28-4.47 \%$ ), $2.43 \%$ (95\% CI: $-0.14-5.06 \%$ ), 2.82\% (95\% CI: $0.09-5.62 \%$ ) and $2.97 \%$ (95\% CI: $0.12-5.91 \%)$, respectively. Of these, lag04 was the most significant $(p=0.041)$. The $\mathrm{PM}_{2.5}$ moving average concentration (lag01and lag02) were marginally significant $(p=0.085 ; p=0.064)$.

See Figure S1 for RR and 95\% CI of mortality per $10 \mu \mathrm{g} / \mathrm{m} 3$ increase in PM2.5 concentration with different lags $0-5$ days prior to mortality (lag0-lag5) and moving averages from day 0 to day prior to mortality (lag01-lag04).

\subsection{Concentration-Response Relationship}

In each single-day lag (lag0-lag5), the strongest effect of $\mathrm{PM}_{2.5}$ concentration upon CVD mortality, IHD mortality and EVD mortality were chosen to make concentration-response relationship figures. See Figure 3 for the concentration-response relationship between $\mathrm{PM}_{2.5}$ daily average concentration and CVD mortality, IHD mortality and EVD mortality with different lags of $\mathrm{PM}_{2.5}$ concentration. According to the figure, based on the control of long-term and seasonal trends of resident mortality, 
effect of day of the week, effect of public holidays, daily average temperature and daily average $\mathrm{RH}$, the relative risk (RR) of CVD mortality, IHD mortality and EVD mortality increases along with the increase in $\mathrm{PM}_{2.5}$ daily average concentration.

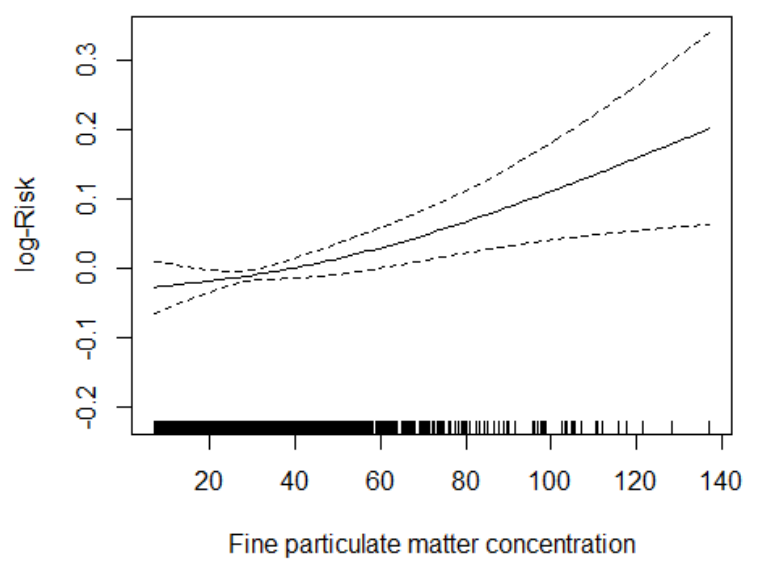

(a)

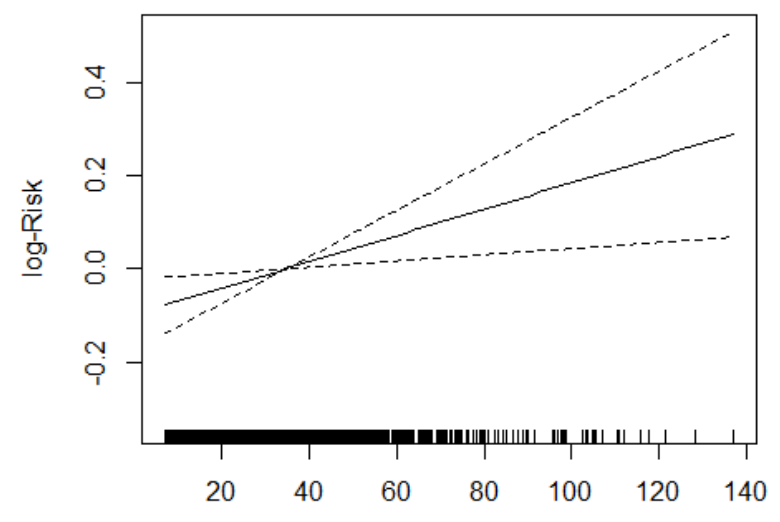

Fine particulate matter concentration

(b)

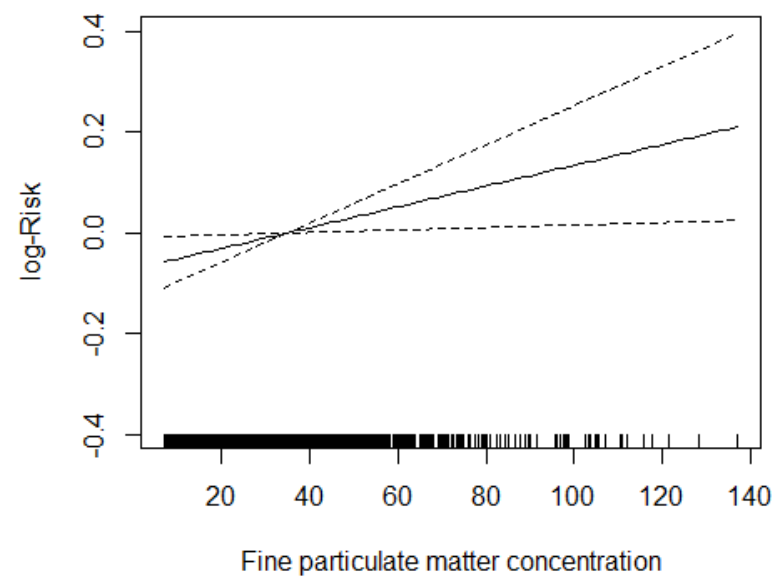

(c)

Figure 3. (a) Association between daily lag5 cardiovascular disease mortality and $\mathrm{PM}_{2.5}$ concentration $\left(\mu \mathrm{g} / \mathrm{m}^{3}\right)$ considered in the generalized additive model (GAM). (b) Association between daily lag2 IHD mortality and $\mathrm{PM}_{2.5}$ concentration $\left(\mu \mathrm{g} / \mathrm{m}^{3}\right)$ considered in the GAM. (c) Association between daily lag4 EVD mortality and $\mathrm{PM}_{2.5}$ concentration $\left(\mu \mathrm{g} / \mathrm{m}^{3}\right)$ considered in the GAM. 


\section{Discussion}

This study aims to explore the association between $\mathrm{PM}_{2.5}$ and CVD mortality, IHD mortality and EVD mortality in areas with lighter air pollution in China. The results show that elevated $\mathrm{PM}_{2.5}$ concentration can increase the risk of CVD mortality, IHD mortality and EVD mortality of residents. There are also lag effects. The effects maintain stability after controlling other pollutants $\left(\mathrm{CO}, \mathrm{O}_{3}\right)$. The results of this study are consistent with the meta-analysis results of previous literature on the relationship between air pollution and CVD mortality $[5,23]$. Epidemiological studies have demonstrated a consistent increased risk for cardiovascular events in relation to both short-term and long-term exposure to present-day concentrations of ambient particulate matter [35].

The putative biological mechanisms linking air pollution to heart disease involve direct effects of pollutants on the cardiovascular system, blood, and lung receptors, and/or indirect effects mediated through pulmonary oxidative stress and inflammatory responses. Direct effects may occur via agents that readily cross the pulmonary epithelium into the circulation, and possibly along with soluble constituents of $\mathrm{PM}_{2.5}$. In addition, activation of pulmonary neural reflexes secondary to PM interactions with lung receptors may play a role. Ensuing alterations in autonomic tone, under appropriate circumstances, might contribute to the instability of a vascular plaque or initiate cardiac arrhythmias. These direct effects of air pollution represent a plausible explanation for the occurrence of rapid cardiovascular responses, such as increased myocardial infarctions. Chronic indirect effects may occur via pulmonary oxidative stress/inflammation induced by inhaled pollutants. This may subsequently contribute to a systemic inflammatory state, which may in turn be capable of activating hemostatic pathways, impairing vascular function, and accelerating atherosclerosis [35].

$\mathrm{PM}_{2.5}$ and daily mortality in 272 Chinese cities from 2013 to 2015 at two levels of nationwide and six geographic regions were studied by Chen et al. The article mainly analyzed two day moving averages of $\mathrm{PM}_{2.5}$ concentrations. The results showed that each $10 \mathrm{mg} / \mathrm{m}^{3}$ increase in the two day moving average of $\mathrm{PM}_{2.5}$ concentrations were significantly associated with increments in mortality of $0.27 \%$ from cardiovascular diseases and $0.23 \%$ from stroke. Furthermore, the associations were stronger in cities with lower $\mathrm{PM}_{2.5}$ levels or higher temperatures [9].

In our study, $\mathrm{PM}_{2.5}$ concentration of lag0-lag5 on CVD mortality had significant effects without controlling other pollutants. When $\mathrm{PM}_{2.5}$ concentration of lag0 to lag5 rose by $10 \mu \mathrm{g} / \mathrm{m}^{3}$, the ER of CVD mortality were $1.60 \%, 1.40 \%, 1.37 \%, 1.00 \%, 0.89 \%$ and $1.50 \%$, respectively. Of these, lag 5 was the most significant, and lag3 and lag4 were marginally significant. According to the meta-analysis results of Atkinson, et al., a $10 \mu \mathrm{g} / \mathrm{m}^{3}$ increment in $\mathrm{PM}_{2.5}$ was associated with a $0.84 \%$ increase in the risk of CVD mortality in the world [5]. The result is lower than the results of our study. Atkinson et al. also reported that the risk of CVD mortality in America, Europe and the Western Pacific region increased by $0.84 \%$, $2.26 \%$ and $0.56 \%$, respectively [5]. The results of our study are between the Americas and European (higher than the results of the study in the Americas and Western Pacific region, lower than European countries). Shenzhen has lighter air pollution and higher temperatures. Chen et al. reported that each $10 \mathrm{mg} / \mathrm{m}^{3}$ increase in two day moving averages of $\mathrm{PM}_{2.5}$ concentrations was significantly associated with increments in mortality of $0.27 \%$ from cardiovascular diseases nationwide [9]. Our study shows that when $\mathrm{PM}_{2.5}$ concentration of lag02 rose by $10 \mu \mathrm{g} / \mathrm{m}^{3}$, the ER of CVD mortality was $2.09 \%$ in Shenzhen. This result is lower than the results of our study. Our findings prove Chen et al.'s discovery. Dai et al. reported a $1.03 \%$ increase in CVD deaths in association with a $10 \mu \mathrm{g} / \mathrm{m}^{3}$ increase in two day averaged $\mathrm{PM}_{2.5}$ concentration [23]. In our study, when $\mathrm{PM}_{2.5}$ concentration of lag02 rose by $10 \mu \mathrm{g} / \mathrm{m}^{3}$, the ER of CVD mortality was $2.09 \%$. Our results are higher than the results of Dai et al. Mate et al. reported that for each increase of $10 \mu \mathrm{g} / \mathrm{m}^{3}$ in daily mean $\mathrm{PM}_{2.5}$ concentration, the relative risks (RR) were as follows: for overall circulatory mortality, associations were established at lags 2 and 6, with a RR of 1.022 and 1.025 respectively in Madrid, Spain [36]. The results are similar to the results of our study. Kan et al. reported that $\mathrm{PM}_{2.5}$ concentration lag0 and moving average concentration lag01 on cardiovascular disease mortality had significant effects in Shanghai, China. A $10 \mu \mathrm{g} / \mathrm{m}^{3}$ increase in the lag01 concentration of $\mathrm{PM}_{2.5}$ corresponded to $0.41 \%$ as the strongest effect increase of cardiovascular 
disease mortality [24]. It is lower than the results of our study (2.09\%). Beelen et al. reported an analysis of 22 European cohorts. For $\mathrm{PM}_{2.5}$, the hazard ratio deaths from cardiovascular disease was 1.21 per $5 \mu \mathrm{g} / \mathrm{m}^{3}$ [37]. Chen et al. reported that stronger associations between $\mathrm{PM}_{2.5}$ exposure and mortality from cardiovascular disease hazard ratio $(\mathrm{HR})=1.35$ in Canada [38]. Pinault et al. reported an analysis of the Canadian community health survey cohort that each $10 \mu \mathrm{g} / \mathrm{m}^{3}$ increase in exposure was associated with increased risks of circulatory disease mortality HR $=1.19$ [39]. Cesaroni et al. reported that the strongest association was found for cardiovascular disease mortality (HR $=1.06$ per $\left.10 \mu \mathrm{g} / \mathrm{m}^{3} \mathrm{PM}_{2.5}\right)$ in Rome [26]. Wong et al. reported that cardiovascular disease mortality hazard ratios per $10 \mu \mathrm{g} / \mathrm{m}^{3}$ increase in $\mathrm{PM}_{2.5}$ was 1.22 in residents older than 65 years in Hong Kong [40]. Zanobett and Schwartz conducted a national, multi-city time-series study of the acute effect of $\mathrm{PM}_{2.5}$ on the increased risk of death for CVD for the years 1999-2005. They found a $0.85 \%$ increase in CVD deaths for a $10 \mu \mathrm{g} / \mathrm{m}^{3}$ increase in two day averaged $\mathrm{PM}_{2.5}$ [41].

In our study, $\mathrm{PM}_{2.5}$ concentration of lag0 to lag2 and lag5 on IHD mortality had significant effects without controlling other pollutants. While $\mathrm{PM}_{2.5}$ concentration of lag0 to lag2 and lag 5 rose by $10 \mu \mathrm{g} / \mathrm{m}^{3}$, the ER of IHD mortality were $2.12 \%, 2.92 \%, 2.87 \%$ and $2.37 \%$, respectively. Of these, lag2 was the most significant $(p=0.009)$. According to the data meta-analysis results of Atkinson et al., a $10 \mu \mathrm{g} / \mathrm{m}^{3}$ increment in $\mathrm{PM}_{2.5}$ was associated with a $3.36 \%$ increase in the risk of IHD mortality in the world [5]. The result is higher than the results of our study. Jerrett et al. used a subset of the American Cancer Society (ACS) cohort (Los Angeles, CA, USA) to estimate a within-city RR of 1.49 per $10 \mu \mathrm{g} / \mathrm{m}^{3}$ increase in $\mathrm{PM}_{2.5}$ [42]. The results are lower than the results of our study. Xie et al. reported that $\mathrm{PM}_{2.5}$ concentration was significantly associated with IHD mortality in Beijing [43]. Our study shows that $\mathrm{PM}_{2.5}$ concentration was marginally significantly associated with IHD mortality. Xie et al. reported that a $10 \mu \mathrm{g} / \mathrm{m}^{3}$ increase in $\mathrm{PM}_{2.5}$ was associated with a $0.27 \%$ increase in IHD mortality on the same day [43]. The result of our study shows that a $10 \mu \mathrm{g} / \mathrm{m}^{3}$ increase in $\mathrm{PM}_{2.5}$ was associated with a $2.12 \%$ increase in IHD mortality on the same day. Chen et al. reported that associations between $\mathrm{PM}_{2.5}$ exposure and IHD mortality $\mathrm{HR}=1.43$ in Canada [38]. Pinault et al. reported that each $10 \mu \mathrm{g} / \mathrm{m}^{3}$ increase in exposure was associated with increased risks of IHD mortality HR $=1.290$ [39]. Cesaroni et al. reported that the strongest association was found for IHD mortality (HR $=1.10$ per $10 \mu \mathrm{g} / \mathrm{m}^{3} \mathrm{PM}_{2.5}$ ) in Rome [26]. Wong et al. reported that IHD mortality hazard ratios per $10 \mu \mathrm{g} / \mathrm{m}^{3}$ increase in $\mathrm{PM}_{2.5}$ were 1.42 in residents older than 65 years Hong Kong [40]. Maté et al. reported that for every increase of $10 \mu \mathrm{g} / \mathrm{m}^{3}$ in daily mean $\mathrm{PM}_{2.5}$ concentration, no statistically significant association was found with other IHD mortality in Madrid, Spain [36]. Maté et al.'s result is different from the results of our study. Thurston et al.'s research shows that associations with IHD mortality varied by $\mathrm{PM}_{2.5}$ mass constituent and source [27].

In our study, $\mathrm{PM}_{2.5}$ concentration of lag0, lag1 and lag3 to lag5 on EVD mortality had significant effects without controlling other pollutants. While $\mathrm{PM}_{2.5}$ concentration of lag0, lag1 and lag3 to lag5 rose by $10 \mathrm{\mu g} / \mathrm{m}^{3}$, the ER of EVD mortality were $2.03 \%, 2.07 \%, 1.90 \%, 2.09 \%$ and $1.99 \%$, respectively. Of these, lag4 was the most significant. The $\mathrm{PM}_{2.5}$ moving average concentration (lag01-lag04) on EVD mortality had significant effects. When $\mathrm{PM}_{2.5}$ concentration of lag01 to lag04 rose by $10 \mu \mathrm{g} / \mathrm{m}^{3}$, the ER of EVD mortality were $2.46 \%, 2.52 \%, 2.78 \%$ and $3.08 \%$, respectively. Of these, lag04 was the most significant. According to the meta-analysis by Wan et al. from 1966-2014 literature, the results show that a $10 \mu \mathrm{g} / \mathrm{m}^{3}$ increase in $\mathrm{PM}_{2.5}$ was associated with a $1.4 \%$ increase in EVD mortality [44]. The result is lower than the results of our study. Chen et al. reported that each $10 \mathrm{mg} / \mathrm{m}^{3}$ increase in two day moving average of $\mathrm{PM}_{2.5}$ concentrations was significantly associated with increments in mortality of $0.23 \%$ from stroke nationwide [9]. Our study shows that when $\mathrm{PM}_{2.5}$ concentration of lag02 rose by $10 \mu \mathrm{g} / \mathrm{m}^{3}$, the ER of EVD mortality were $2.52 \%$ in Shenzhen. Our results confirmed the discovery by Chen et al. that the associations between $\mathrm{PM}_{2.5}$ and daily mortality were stronger in cities with lower $\mathrm{PM}_{2.5}$ levels or higher temperatures [9]. Pinault et al. reported an analysis of the Canadian community health survey cohort that each $10 \mu \mathrm{g} / \mathrm{m}^{3}$ increase in exposure was associated with increased risks of EVD mortality HR $=1.241$ (significant HR $p>0.05$ ) [39]. Wong et al. reported 
that EVD mortality hazard ratios per $10 \mu \mathrm{g} / \mathrm{m}^{3}$ increase in $\mathrm{PM}_{2.5}$ was 1.24 in residents older than 65 years in Hong Kong [40]. Maté et al. reported that for every increase of $10 \mu \mathrm{g} / \mathrm{m}^{3}$ in daily mean $\mathrm{PM}_{2.5}$ concentration, no statistically significant association was found with EVD mortality in Madrid, Spain [36]. Mate et al.'s result is different from the results of our study.

The time series chart showed that the variation trend of cardiovascular disease mortality counts and $\mathrm{PM}_{2.5}$ concentration basically matched. According to the concentration-response relationship figures, the RR of cardiovascular disease mortality, IHD mortality and EVD mortality increased along with the increase of $\mathrm{PM}_{2.5}$ daily average concentration. The time series chart and the concentration-response relationship figures all indicate that $\mathrm{PM}_{2.5}$ has an effect on the death from cardiovascular disease of the residents.

The average age of death from cardiovascular disease was 67.4 years old in Shenzhen during 2013-2015; the average age of IHD death was 66.6 years old; the average age of EVD death was 66.0 years old. The results are younger than China's average life expectancy (the average life expectancy of China's population is 74.8 years old) [45]. The reason for this may be that although Shenzhen belongs to the economically developed area, there is a higher standard of living, but Shenzhen is a city of immigrants. Many people come from all over the country to live and work in Shenzhen, these people are mainly young, so the residents of Shenzhen's actual age is younger than that in other parts of China. This is also one of the reasons why the annual mortality rate in Shenzhen is lower than that in other parts of China. The death rate is 2.4 per thousand in 2014 in Shenzhen, and China is 7.2 per thousand in $2014[46,47]$.

Reported results at different places in the world are not exactly the same, which may be related to the following factors. First, the main source of pollutants, pollutant concentration and composition of $\mathrm{PM}_{2.5}$ are different in different areas and different periods. Ambient particulate matter is a heterogeneous mixture of various compounds (e.g., organic and elemental carbon, metals, sulfates, nitrates, and microorganisms) from multiple sources (e.g., traffic, manufacturing, power generation) [44]. Second, different methods were used to assess residents' exposure to pollutants. Third, the control confounding factors in the process of study are not completely the same. Fourth, the definitions of ICD coding of diseases classification are different. Fifth, the study populations have different demographic characteristics. Sixth, the meteorological factors of research in different areas and different periods are different. Seventh, small study biases exist in studies of cardiovascular disease mortality [5]. Reasons for heterogeneity in effect estimates in different regions of the world require further investigation $[5,44]$.

\section{Strengths and Limitations}

In this study, data on resident mortality were collected from Shenzhen CDC, Shenzhen Public Security Bureau and Shenzhen Funeral Home, which represent deaths of local residents. This study analyzed the data of 2013-2015 for three consecutive years, and the results were stable. In this study, several factors may affect the mortality of residents, such as long-term trend, seasonal trend, day of the week, public holiday, daily average temperature and daily average relative humidity, were controlled in the model. The model objectively analyzes the relationship between $\mathrm{PM}_{2.5}$ concentration and total cardiovascular disease mortality, $\mathrm{PM}_{2.5}$ concentration and IHD mortality, $\mathrm{PM}_{2.5}$ concentration and EVD mortality.

The present study also has some limitations. The local-specific factors that could affect the association between pollutant exposure and mortality such as PM chemical composition, existing emission sources, socioeconomic factors, etc. are not incorporated in the study and need to be researched further.

\section{Conclusions}

Although the mean of $\mathrm{PM}_{2.5}$ daily average concentration was $35.0 \mu \mathrm{g} / \mathrm{m}^{3}$ in Shenzhen, showing a slight pollution in China, $\mathrm{PM}_{2.5}$ still increased the amount of cardiovascular disease, IHD and EVD 
mortality. Therefore, it is recommended that residents need to use personal protection, especially the residents with cardiovascular disease and in pollution weather, thereby reducing the adverse effects of $\mathrm{PM}_{2.5}$ on health. The government still needs to strengthen the governance of air pollution in areas with lighter air pollution.

Supplementary Materials: The following are available online at http:/ / www.mdpi.com/1660-4601/15/9/1918/ s1, 1. Analysis of GAM (Figure S1. RR and $95 \%$ CI of mortality per $10 \mu \mathrm{g} / \mathrm{m}^{3}$ increase in $\mathrm{PM}_{2.5}$ concentration with different lags $0-5$ days prior to mortality (lag0-lag5) and moving averages from day 0 to day prior to mortality (lag01-lag04)). 2. People's Republic of China Ministry of Environmental Protection, Atmospheric environmental quality standards (GB3095-2012) (Table S1).

Author Contributions: J.C. (Junfang Cai) and S.Y. analyzed the data and wrote the draft of the paper. Y.P. critically revised the manuscript for important intellectual content analyzed the data. C.P. and Y.L. collected the data. N.L. and J.J. participated in the interpretation of the findings. J.C. (Jinquan Cheng) conceived and designed the study. All authors read and approved the final manuscript.

Acknowledgments: The authors thank Chew Ginger L.; Yip Fuyuen Y. and George A. Conway of US CDC for their invaluable guidance, comments and language revision of the manuscript. The authors thank Jinghua Cai of University of Southern California for language revision of the manuscript.

Conflicts of Interest: The authors declare no conflict of interest.

\section{References}

1. Jiménez, E.; Linares, C.; Rodríguez, L.F.; Bleda, M.J.; Díaz, J. Short-term impact of particulate matter $\left(\mathrm{PM}_{2.5}\right)$ on daily mortality among the over-75 age group in Madrid (Spain). Sci. Total Environ. 2009, 407, 5486-5492. [CrossRef] [PubMed]

2. Ito, K.; Mathes, R.; Ross, Z.; Nadas, A.; Thurston, G.; Matte, T. Fine particulate matter constituents associated with cardiovascular hospitalizations and mortality in New York City. Environ. Health Perspect. 2011, 119, 467-473. [CrossRef] [PubMed]

3. Ostro, B.; Feng, W.Y.; Broadwin, R.; Green, S.; Lipsett, M. The effects of components of fine particulate air pollution on mortality in California: Results from CALFINE. Environ. Health Perspect. 2007, 115, 13-19. [CrossRef] [PubMed]

4. Zhou, J.; Ito, K.; Lall, R.; Lippmann, M.; Thurston, G. Time-series analysis of mortality effects of fine particulate matter components in Detroit and Seattle. Environ. Health Perspect. 2011, 119, 461-466. [CrossRef] [PubMed]

5. Atkinson, R.W.; Kang, S.; Anderson, H.R.; Mills, I.C.; Walton, H.A. Epidemiological time series studies of $\mathrm{PM}_{2.5}$ and daily mortality and hospital admissions: A systematic review and meta-analysis. Thorax 2014, 69, 660-665. [CrossRef] [PubMed]

6. The Report of the 19th Session of National Congress of the Communist Party of China, China, 18 October to 24 October 2017. Available online: http:/ / www.lsctgz.com/article/djzl/dzz/201710/1746.html (accessed on 21 July 2018).

7. Ministry of Ecology and Environment of the People's Republic of China. Chinese Environmental Status Bulletin. Available online: http:/ /www.zhb.gov.cn/hjzl/zghjzkgb/lnzghjzkgb/ (accessed on 21 July 2018).

8. National Air Quality Real-time Ranking. Available online: http://www.86pm25.com/paiming.htm (accessed on 21 July 2018).

9. Chen, R.J.; Yin, P.; Meng, X.; Liu, C.; Wang, L.J.; Xu, X.H.; Ross, J.A.; Tse, L.A.; Zhao, Z.H.; Kan, H.D.; et al. Fine Particulate Air Pollution and Daily Mortality. A Nationwide Analysis in 272 Chinese Cities. Am. J. Respir. Crit. Care Med. 2017, 196, 73-81. [CrossRef] [PubMed]

10. Bell, M.L.; Peng, R.D.; Dominici, F.; Samet, J.M. Emergency hospital admissions for cardiovascular diseases and ambient levels of carbon monoxide: Results for 126 United States urban counties, 1999-2005. Circulation 2009, 120, 949-955. [CrossRef] [PubMed]

11. Mazidi, M.; Speakman, J.R. Impact of Obesity and Ozone on the Association between Particulate Air Pollution and Cardiovascular Disease and Stroke Mortality among US Adults. J. Am. Heart Assoc. 2018, 7, 1-14. [CrossRef] [PubMed]

12. Hastie, T.; Tibshirani, R. Generalized additive models for medical research. Stat. Methods Med. Res. 1995, 4, 187-196. [CrossRef] [PubMed] 
13. Schwartz, J.; Laden, F.; Zanobetti, A. The Concentration-Response Relation between $\mathrm{PM}_{2.5}$ and Daily Deaths. Environ. Health Perspect. 2002, 110, 1025-1029. [CrossRef] [PubMed]

14. Schwartz, J. Air pollution and daily mortality: A review and meta-analysis. Environ. Res. 1994, 64, 36-52. [CrossRef] [PubMed]

15. Hoek, G.; Schwartz, J.; Groot, B.; Eilers, P. Effects of ambient particulate matter and ozone on daily mortality in Rotterdam, The Netherlands. Arch. Environ. Health 1997, 52, 455-463. [CrossRef] [PubMed]

16. Michelozzi, P.; Forastiere, F.; Fusco, D.; Perucci, C.A.; Ostro, B.; Ancona, C.; Pallotti, G. Air pollution and daily mortality in Rome, Italy. Occup. Environ. Med. 1998, 55, 605-610. [CrossRef] [PubMed]

17. Katsouyanni, K.; Touloumi, G.; Samoli, E.; Gryparis, A.L.; Tertre, A.; Monopolis, Y.; Rossi, G.; Zmirou, D.; Ballester, F.; Boumghar, A.; et al. Confounding and effect modification in the short-term effects of ambient particles on total mortality: Results from 29 European Cities within the APHEA2 Project. Epidemiology 2001, 12, 521-531. [CrossRef] [PubMed]

18. Ver Hoef, J.M.; Boveng, P.L. Quasi-Poisson vs.negative binomial regression: How should we model overdispersed count data? Ecology 2007, 88, 2766-2772. [CrossRef] [PubMed]

19. Kloog, I.; Ridgway, B.; Koutrakis, P.; Coull, B.A.; Schwartz, J.D. Long-and short-term exposure to $\mathrm{PM}_{2.5}$ and mortality: Using novel exposure models. Epidemiology 2013, 24, 555-561. [CrossRef] [PubMed]

20. Qiu, H.; Yu, I.T.; Tian, L.; Wang, X.; Tse, L.A.; Tam, W.; Wong, T.W. Effects of Coarse Particulate Matter on Emergency Hospital Admissions for Respiratory Diseases: A Time-Series Analysis in Hong Kong. Environ. Health Perspect. 2012, 120, 572-676. [CrossRef] [PubMed]

21. Wong, T.W.; Tam, W.; Tak Sun Yu, I.; Wun, Y.T.; Wong, A.H.; Wong, C.M. Association between air pollution and general practitioner visits for respiratory diseases in Hong Kong. Thorax 2006, 61, 585-591. [CrossRef] [PubMed]

22. Wood, S.N. Generalized Additive Models: An Introduction with R; CRC Press: Boca Raton, FL, USA, 2006; Available online: https://www.crcpress.com/Generalized-Additive-Models-An-Introduction-with-R/ Wood/9781584884743 (accessed on 5 August 2015).

23. Dai, L.Z.; Zanobetti, A.; Koutrakis, P.; Schwartz, J.D. Associations of Fine Particulate Matter Species with Mortality in the United States: A Multicity Time-Series Analysis. Environ. Health Perspect. 2014, 122, 837-842. [CrossRef] [PubMed]

24. Kan, H.D.; London, S.J.; Chen, G.H.; Zhang, Y.H.; Song, G.X.; Zhao, N.Q.; Jiang, L.L.; Chen, B. Differentiating the effects of fine and coarse particles on daily mortality in Shanghai, China. Environ. Int. 2007, 33, 376-384. [CrossRef] [PubMed]

25. Shi, L.H.; Zanobetti, A.; Kloog, I.; Coull, B.A.; Koutrakis, P.; Melly, S.J.; Schwartz, J.D. Low-Concentration $\mathrm{PM}_{2.5}$ and Mortality: Estimating Acute and Chronic Effects in a Population-Based Study. Environ. Health Perspect. 2016, 124, 46-52. [CrossRef] [PubMed]

26. Cesaroni, G.; Badaloni, C.; Gariazzo, C.; Stafoggia, M.; Sozzi, R.; Davoli, M.; Forastiere, F. Long-Term Exposure to Urban Air Pollution and Mortality in a Cohort of More than a Million Adults in Rome. Environ. Health Perspect. 2013, 121, 324-331. [CrossRef] [PubMed]

27. Thurston, G.D.; Burnett, R.T.; Turner, M.C.; Shi, Y.; Krewski, D.; Lall, R.; Ito, K.; Jerrett, M.; Gapstur, S.M.; Diver, W.R.; et al. Ischemic Heart Disease Mortality and Long-Term Exposure to Source-Related Components of U.S. Fine Particle Air Pollution. Environ. Health Perspect. 2016, 124, 785-794. [CrossRef] [PubMed]

28. Cifuentes, L.A.; Vega, J.; Köpfer, K.; Lave, L.B. Effect of the Fine Fraction of Particulate Matter versus the Coarse Mass and Other Pollutants on Daily Mortality in Santiago, Chile. J. Air Waste Manag. Assoc. 2000, 50, 1287-1298. [CrossRef] [PubMed]

29. Krall, J.R.; Anderson, G.B.; Dominici, F.; Bell, M.L.; Peng, R.D. Short-term Exposure to Particulate Matter Constituents and Mortality in a National Study of U.S. Urban Communities. Environ. Health Perspect. 2013, 121, 1148-1153. [CrossRef] [PubMed]

30. Bell, M.L.; Samet, J.M.; Dominici, F. Time-series studies of particulate matter. Annu. Rev. Public Health 2004, 25, 247-280. [CrossRef] [PubMed]

31. Roberts, S. A new model for investigating the mortality effects of multiple air pollutants in air pollution mortality time-series studies. J. Toxicol. Environ. Health A 2006, 69, 417-435. [CrossRef] [PubMed]

32. Zhang, Y.H.; Huang, W.; London, S.J.; Song, G.X.; Chen, G.H.; Jiang, L.L.; Zhao, N.Q.; Chen, B.H.; Kan, H.D. Ozone and daily mortality in Shanghai, China. Environ. Health Perspect. 2006, 114, 1227-1232. [CrossRef] [PubMed] 
33. Janssen, N.A.; Fischer, P.; Marra, M.; Ameling, C.; Cassee, F.R. Short-term effects of $\mathrm{PM}_{2.5}$, $\mathrm{PM}_{10}$ and $\mathrm{PM}_{2.5-10}$ on daily mortality in the Netherlands. Sci. Total Environ. 2013, 463-464, 20-26. [CrossRef] [PubMed]

34. Ministry of Environmental Protection of the People's Republic of China. Ambient Air Quality Standards. GB3095-2012. Available online: http:/ / kjs.mep.gov.cn/hjbhbz/bzwb/dqhjbh/dqhjzlbz/201203/t20120302_ 224165.htm (accessed on 6 February 2018).

35. Brook, R.D.; Franklin, B.; Cascio, W.; Hong, Y.; Howard, G.; Lipsett, M.; Luepker, R.; Mittleman, M.; Samet, J.; Smith, S.C., Jr.; et al. Air Pollution and Cardiovascular Disease: A Statement for Healthcare Professionals from the Expert Panel on Population and Prevention Science of the American Heart Association. Circulation 2004, 109, 2655-2671. [CrossRef] [PubMed]

36. Maté, T.; Guaita, R.; Pichiule, M.; Linares, C.; Díaz, J. Short-term effect of fine particulate matter $\left(\mathrm{PM}_{2.5}\right)$ on daily mortality due to diseases of the circulatory system in Madrid (Spain). Sci. Total Environ. 2010, 408, 5750-5757. [CrossRef] [PubMed]

37. Beelen, R.; Stafoggia, M.; Raaschou-Nielsen, O.; Andersen, Z.J.; Xun, W.W.; Katsouyanni, K.; Dimakopoulou, K.; Brunekreef, B.; Weinmayr, G.; Hoffmann, B.; et al. Long-term exposure to air pollution and cardiovascular mortality: An analysis of 22 European cohorts. Epidemiology 2014, 25, 368-378. [CrossRef] [PubMed]

38. Chen, H.; Burnett, R.T.; Copes, R.; Kwong, J.C.; Villeneuve, P.J.; Goldberg, M.S.; Brook, R.D.; van Donkelaar, A.; Jerrett, M.; Martin, R.V.; et al. Ambient Fine Particulate Matter and Mortality among Survivors of Myocardial Infarction: Population-Based Cohort Study. Environ. Health Perspect. 2016, 124, 1421-1428. [CrossRef] [PubMed]

39. Pinault, L.; Tjepkema, M.; Crouse, D.L.; Weichenthal, S.; van Donkelaar, A.; Martin, R.V.; Brauer, M.; Chen, H.; Burnett, R.T. Risk estimates of mortality attributed to low concentrations of ambient fine particulate matter in the Canadian community health survey cohort. Environ. Health 2016, 11, 15-18. [CrossRef] [PubMed]

40. Wong, C.M.; Lai, H.K.; Tsang, H.; Thach, T.Q.; Thomas, G.N.; Lam, K.B.; Chan, K.; Yang, L.; Lau, A.K.; Ayres, J.G.; et al. Satellite-Based Estimates of Long-Term Exposure to Fine Particles and Association with Mortality in Elderly Hong Kong Residents. Environ. Health Perspect. 2015, 123, 1167-1172. [CrossRef] [PubMed]

41. Zanobetti, A.; Schwartz, J. The effect of fine and coarse particulate air pollution on mortality: A national analysis. Environ. Health Perspect. 2009, 117, 898-903. [CrossRef] [PubMed]

42. Jerrett, M.; Burnett, R.T.; Ma, R.; Pope, C.A., 3rd; Krewski, D.; Newbold, K.B.; Thurston, G.; Shi, Y.; Finkelstein, N.; Calle, E.E.; et al. Spatial analysis of air pollution and mortality in Los Angeles. Epidemiology 2005, 16, 727-736. [CrossRef] [PubMed]

43. Xie, W.; Li, G.; Zhao, D.; Xie, X.; Wei, Z.; Wang, W.; Wang, M.; Li, G.; Liu, W.; Sun, J.; et al. Relationship between fine particulate air pollution and ischaemic heart disease morbidity and mortality. Heart 2015, 101, 257-263. [CrossRef] [PubMed]

44. Wang, Y.; Eliot, M.N.; Wellenius, G.A. Short-term Changes in Ambient Particulate Matter and Risk of Stroke: A Systematic Review and Meta-analysis. J. Am. Heart Assoc. 2014, 3. [CrossRef] [PubMed]

45. National Bureau of Statistics of the People's Republic of China. TABULATION ON THE 2010 POPULATION CENSUS OF THE PEOPLES REPUBLIC OF CHINA. Available online: http:/ / www.stats.gov.cn/tjsj/pcsj/ rkpc/6rp/indexch.htm (accessed on 6 February 2018).

46. SHENZHEN CHINA. Shenzhen Statistics. SHENZHEN STAISTICAL YEARBOOK. Available online: http: / / www.sztj.gov.cn/xxgk/tjsj/tjnj/201606/W020160607351981905550.pdf (accessed on 6 February 2018).

47. National Bureau of Statistics of the People's Republic of China. CHINA STAISTICAL YEARBOOK. Available online: http://www.stats.gov.cn/tjsj/tjcbw/201512/t20151210_1287840.html (accessed on 6 February 2018).

(C) 2018 by the authors. Licensee MDPI, Basel, Switzerland. This article is an open access article distributed under the terms and conditions of the Creative Commons Attribution (CC BY) license (http://creativecommons.org/licenses/by/4.0/). 